\title{
Article
}

\section{Posttraumatic stress symptoms mediate the relationship between adverse childhood experiences, avoidant personality traits and resilience}

Rawlins, Bethan, Brooks, Matthew and Khan, Roxanne

Available at http://clok.uclan.ac.uk/32889/

Rawlins, Bethan, Brooks, Matthew and Khan, Roxanne ORCID: 0000-00023485-2450 (2020) Posttraumatic stress symptoms mediate the relationship between adverse childhood experiences, avoidant personality traits and resilience. Anxiety, Stress \& Coping: An International Journal, 33 (5). pp. 590 601. ISSN 1061-5806

It is advisable to refer to the publisher's version if you intend to cite from the work. http://dx.doi.org/10.1080/10615806.2020.1768532

For more information about UCLan's research in this area go to http://www.uclan.ac.uk/researchgroups/ and search for <name of research Group>.

For information about Research generally at UCLan please go to http://www.uclan.ac.uk/research/

All outputs in CLoK are protected by Intellectual Property Rights law, including Copyright law. Copyright, IPR and Moral Rights for the works on this site are retained by the individual authors and/or other copyright owners. Terms and conditions for use of this material are defined in the policies page. 
RUNNING HEAD: Traumatic stress, avoidant personality, resilience

Suggested citation: Rawlins, B., Brooks, M. \& Khan, R. (2020, accepted). Posttraumatic stress as a mediator of the relationship between adverse childhood experiences, avoidant personality traits and resilience. Anxiety, Stress \& Coping

For a copy of the published article, please email rkhan2@uclan.ac.uk

\title{
Posttraumatic stress symptoms mediate the relationship between adverse childhood experiences, avoidant personality traits and resilience
}

\author{
Bethan Rawlins, MSc \\ University of Central Lancashire, School of Psychology, UK \\ Matthew Brooks, PhD* \\ Manchester Metropolitan University, Department of Psychology, UK
}

ORCID: 0000-0002-5469-7769

Roxanne Khan, PhD

University of Central Lancashire, School of Psychology, UK

ORCID: 0000-0002-3485-2450

*Corresponding Author:

Dr Matthew Brooks

Department of Psychology

Brooks Building

Manchester Metropolitan University

Manchester

M15 6GX

United Kingdom

Email: $\underline{\text { m.brooks@mmu.ac.uk }}$ 
RUNNING HEAD: Traumatic stress, avoidant personality, resilience

\begin{abstract}
Background and Objectives: It is widely recognized that posttraumatic stress (PTS) symptoms, resulting from adverse childhood experiences (ACEs), have myriad detrimental effects on the wellbeing of sufferers. Yet the extent to which PTS symptoms mediate positive and negative outcomes is less well-known. This study therefore explored whether PTS symptoms indirectly explain avoidant personality disorder (AVPD) traits and resilience following multiple ACEs.
\end{abstract}

Design: A correlational design using mediation analyses was conducted.

Method: One-hundred and seventy-seven participants (68.4\% female; aged 18-73 years) completed an online survey measuring experiences of childhood adversity, AVPD traits, resilience, and PTS symptoms.

Results: The pathway from ACEs to AVPD mediated by PTS symptoms was both positive and significant. The indirect relationship from ACEs to resilience was also significantly and negatively mediated by PTS symptoms. Of the PTS dimensions, avoidance and hyperarousal mediated the relationship between the number of ACEs and AVPD traits, while intrusions mediated the association between the number of ACEs and resilience.

Conclusions: These findings indicate that PTS symptoms can have both detrimental and adaptive effects on psychological wellbeing. Treatment and management of PTS symptoms is therefore key to the improvement of overall psychological functioning following ACEs.

Keywords: Adverse childhood experiences, avoidant personality, mediation, posttraumatic stress, resilience

Disclosure of interest: The authors report no conflict of interest. 
RUNNING HEAD: Traumatic stress, avoidant personality, resilience

\section{Introduction}

An increased awareness of adverse childhood experiences (ACEs) has motivated research efforts to better understand its psychological consequences. Studies indicate that multiple ACEs (e.g., emotional, physical and sexual abuse or neglect, and family dysfunction) are likely to co-occur, therefore, it is unsurprising that they are associated with negative physical, psychological and social functioning (Bellis, Lowey, Leckenby, Hughes, \& Harrison, 2014; Finkelhor et al., 2011). For instance, studies have linked the risk of developing depression (Chapman et al., 2004), substance misuse (Bellis et al., 2017) and posttraumatic stress (PTS) symptoms (Schalinski et al., 2016) with cumulative ACEs. Further research into the psychological impact of ACEs is needed to inform treatment efforts to help guide survivors' recovery from significant challenges in early life.

PTS symptoms that result from ACEs are often characterized by intrusive thoughts, avoiding memories of the experience, hyperarousal, and negative changes in cognition and mood (Karam et al., 2014; Schalinski et al., 2016). In some cases, these symptoms are chronic and long-lasting, persisting into adulthood (Bellis et al., 2014; Chapman et al., 2004; Cook et al., 2017; Felitti et al., 1998; Finkelhor et al., 2011). Trauma theories have traditionally proposed that PTS symptoms and other pathological symptoms represent a failure to cognitively process adverse experiences and encode trauma memories (Halligan, Michael, Clark, \& Ehlers, 2003). More recent interpretations, however, posit that PTS symptoms are natural reaction to traumatic events, and can actually serve as a catalyst for positive outcomes, including posttraumatic growth (Brooks, Graham-Kevan, Robinson, \& Lowe, 2019), and resilience (Bensimon, 2012).

Numerous explanations have been proposed as to the detrimental effect of cumulative ACEs on psychological functioning. One view is that symptom complexity increases as a 
RUNNING HEAD: Traumatic stress, avoidant personality, resilience

function of the frequency and magnitude of exposure to adverse life events (Neuner et al., 2004; Schalinski et al., 2016). These changes occur within a 'dose-response' relationship, with more frequent exposures associated with poorer outcomes. For instance, individuals reporting multiple ACEs are more likely to endorse symptoms of PTS, guilt, dissociation, shame and interpersonal difficulties compared to those who experience an isolated event (Hagenaars, Fisch, \& van Minnen, 2011; Sulliman et al., 2009). A separate yet complimentary perspective argues that the timing of events is critical to subsequent psychological outcomes. Adverse events occurring at a critical developmental time in childhood can disrupt the formation of secure attachments (Cook et al., 2017), manifesting in a poor sense of self-concept (Turner, Shattuck, Finkelhor, \& Hamby, 2017), emotion regulation difficulties (Stevens et al., 2013), and increased depression and anxiety compared to people who experience adversity in adulthood (Dunn, Nishimi, Powers, \& Bradley, 2017), all of which are regarded as markers of healthy psychological functioning (Cook et al., 2017). Collectively, the experience of frequent and early life adversity can be indicative of complex trauma presentations (Cook et al., 2017), which may present additional psychological challenges for survivors compared to individuals without ACEs.

While these explanations have been proposed to account for differential psychological outcomes following ACEs, people do not respond to adverse events identically (Seery, Holman, \& Silver, 2010). Indeed, studies that focus on the objective characteristics of the event, such as the frequency and timing of the trauma, reveal that they do not always explain the degree of distress experienced (Brooks et al., 2019). Rather, it is likely that mediating factors can determine why some people report more or less distress than others following ACEs. Despite well-documented associations between childhood adversity and PTS, less research has examined how PTS symptoms indirectly explain relationships with other outcomes that arise following ACEs. This has included PTS symptoms as a mediator of self- 
RUNNING HEAD: Traumatic stress, avoidant personality, resilience

reported quality of life (Hu, Xu, Liu, 2018) and adolescent interpersonal revictimisation in females (Auslander, Myers Tlapek, Threlfall, Edmond \& Dunn, 2015). Wider evidence is limited, as existing studies tend to focus on direct predictors of PTS symptoms (Elklit, Christiansen, Palic, Karsberg, \& Eriksen, 2014), or focus on other mediators of negative outcomes following ACEs, such as rumination on anxiety symptoms (Kim, Jin, Jung, Hahn, \& Lee, 2017). In addition, some existing ACE studies address only one type of childhood trauma such as emotional abuse (Goodman, Gutarra, Billingsley, Keiser, \& Gitari, 2017) rather than accounting for the number of different types of ACEs that people may experience. As PTS symptoms are often implicated in people's responses to childhood adversity (Karam et al., 2014; Schalinski et al., 2016), there is a need to further understand the potential range of outcomes that can arise from their presence to inform treatment efforts.

Posttraumatic stress as a mediator of avoidant personality disorder traits and resilience following ACEs

Within discussions of PTS development following ACEs, a large body of literature has examined the relationship between childhood trauma and borderline personality disorder (BPD; e.g. Ball \& Links, 2009; MacIntosh, Godbout, \& Dubash, 2015), although less research has addressed the pathways whereby avoidant personality disorder (AVPD) traits arise following ACEs. Characterized by a fear of abandonment, hypersensitivity to negative evaluation and social inhibition, AVPD traits are thought to arise through rejection and neglect from caregivers (Lampe \& Malhi, 2018; Rettew et al., 2003). This can be compounded with ACEs to worsen the chronicity of other outcomes, such as depression (Klein, Roniger, Schweiger, Spath \& Brodbeck, 2015) and self-harm (Gratz \& Tull, 2012). In response to inaccessible or inconsistent parenting, children may develop avoidance and hypervigilance as coping strategies which then extend to other social situations. Some research indicates that ACEs may be positively related to AVPD traits (Hageman, Francis, 
RUNNING HEAD: Traumatic stress, avoidant personality, resilience

Field, \& Carr, 2015), although no studies currently examine mediators that explain why some people report AVPD traits and others do not.

While studies are few, research has indicated possible links between PTS symptoms and AVPD traits. For instance, AVPD traits may arise following exacerbated PTS symptoms (Bovin, Wolf, \& Resick, 2017), and determine treatment responsivity within trauma-specific interventions targeting reductions in PTS (Békés, Beaulieu-Prévost, Guay, Belleville, \& Marchand, 2018). Other research has found that self-reported ACEs are associated with increased AVPD symptoms in adulthood (Hageman, Francis, Field, \& Carr, 2015), which suggests that childhood experiences could be pertinent in the development of AVPD traits. Together these findings may explain why it is difficult for people with AVPD traits to recover from adverse experiences, although further research is needed. It is proposed that ACEs perpetrated by a caregiver creates a sense of 'betrayal' that conflicts with the young person's reliance on that caregiver for their protection (Freyd, 1994). As a result, survivors may maintain a sense of avoidance as a protective mechanism to maintain survival, rather than to mitigate distress.

Separately, research has started to address positive outcomes that may arise following ACEs, such as resilience (e.g. Banyard, Hamby, \& Grych, 2017; Carlson et al., 2016). Resilience has been identified as one possible indicator of recovery following ACEs, and is generally construed as the ability to adapt well and 'bounce back' in the face of adversity (Smith et al., 2008). The concept is dynamic in nature and universally agreed definitions are lacking; indeed, some research infers resilience through composite factors, such as social support and a reduction (or absence) of pathology (e.g. Domhardt, Münzer, Fegert, \& Goldbeck, 2015). Broadly, resilience can be viewed as a short-term response to trauma or distress (Bonnano, 2004), a process of positive adaptation from the struggle with adverse events (Olsson, Jerneck, Thoren, Persson \& O’Byrne, 2015), or favorable outcomes and 
RUNNING HEAD: Traumatic stress, avoidant personality, resilience

competency following adversity (Masten, 2001). Notwithstanding differences in conceptualization, resilience appears to be an important construct that could serve as a marker of psychological functioning after ACEs.

There is no existent research that has directly assessed the impact of multiple ACEs on resilience, although some studies have explored the influence of childhood trauma more generally. In a study of predictors of resilience in a community sample, childhood maltreatment only independently explained an additional two percent of variance in resilience scores compared to $11 \%$ for demographic factors (Campbell-Sills, Forde, \& Stein, 2009). This finding suggests that the impact of childhood trauma on levels of resiliency is relatively modest, which is supported by arguments that most people are remarkably resilient in the face of cumulative adversity (Seery et al., 2010). It may be that resilience serves as a buffer against distress of ACEs, enhancing wellbeing and reducing PTS symptoms (Lee, Ahn, Jeong, Chae, \& Choi, 2014; Pietrzak et al., 2010; Sexton, Hamilton, McGinnis, Rosenblum, \& Muzik, 2015). Given these limited findings, more research is needed to explore how PTS symptoms impact on resilience following ACEs. As there is a continued focus on promoting resilience within trauma-focused therapies (Happer, Brown, \& Sharma-Patel, 2017), further research into the role of PTS symptoms in explaining resilient outcomes following ACEs is warranted.

\section{Aims of study}

The aim of this study is to investigate PTS symptoms as a mechanism whereby some people may report more (or less) AVPD traits and resiliency than others following ACEs. Based on prior literature, it is expected that cumulative ACEs alone will not explain AVPD traits, however, the number of ACEs experienced will result in more PTS symptoms, which will increase the severity of AVPD traits. Due to limited research on multiple ACEs and 
RUNNING HEAD: Traumatic stress, avoidant personality, resilience

resilience, no directional hypotheses were made. Furthermore, the intent of the study is to examine patterns of findings in a non-clinical sample, rather than seek to apply diagnostic criteria for AVPD or PTS.

\section{Method}

\section{Participants}

One hundred and seventy-seven participants (68.4\% female) aged from 18 to 73 years $(M=33.56, S D=13.42)$ were recruited via opportunity sampling using social media sites, and advertisement posters around a university campus in North West England. The distribution of ACEs self-reported by participants is presented in Table 1. The most widely experienced ACEs which were reported to have occurred at least once or more times were bullying (79.7\%), being hit with a blunt or sharp object (66.1\%), being yelled, screamed, sworn at, insulted or humiliated (63.4\%), and witnessing a parent, caregiver or other household member being physically attacked (63.3\%). In addition, 15.8\% of respondents indicated that their parents or guardians did not understand their problems or worries. Less than a fifth of the sample (18.6\%) believed their parents or guardians knew where they were during their free time outside of school or work. Literature has indicated that exposure to four or more ACEs is associated with negative physical and psychological outcomes (e.g. Crouch, Radcliff, Strompolis, \& Srivastav, 2019). In this sample, 12 participants (10.2\%) reported one ACE, while over three-quarters (77.4\%) experienced four or more ACEs.

[INSERT TABLE 1 ABOUT HERE]

\section{Measures}

Adverse childhood experiences. The Adverse Childhood Events- International Questionnaire (ACE-IQ; World Health Organisation, 2012) was used to measure adverse 
RUNNING HEAD: Traumatic stress, avoidant personality, resilience

experiences in childhood before age 18. The ACE-IQ was modified slightly for the purposes of obtaining the number of ACEs. Questions regarding warfare-related experiences were removed as they were deemed irrelevant for the majority of likely participants. Furthermore, the four original questions about sexual abuse were condensed into one less explicit version, and wording surrounding weapon use was altered in some of the physical abuse questions to minimize negative effects on participants. ACE prevalence was assessed using 21 items measured on a four-point scale $(0=$ never to $3=$ many times $)$, with items such as, 'did someone touch you or make you touch them in a sexual way when you did not want them to?' The ACE-IQ demonstrated good reliability in this study $(\alpha=.88)$.

Posttraumatic stress symptoms. The PTSD Checklist for DSM-V (PCL-5; Weathers et. al, 2013) is a 20-item screening measure of PTS symptoms according to the DSM-V. It consists of four subscales (avoidance, hyperarousal, intrusions and negative cognitions) as well as a combined overall score. For every item such as, 'How much have you been bothered by feeling jumpy or easily startled?', respondents rate the severity of each symptom on a five-point scale $(0=$ not at all to $4=$ extremely $)$ in the past month. A total score is obtained, with higher scores reflecting greater distress. Weathers et al. (2013) suggest a score of 31-33 and above indicates probable PTSD, although further research is needed. On this basis, $29.9 \%$ of study participants would meet this threshold. The PCL-5 demonstrates high internal consistency and test-retest reliability (Bovin et al., 2016), which was replicated in the current study for the overall PTS score $(\alpha=.95)$, and the four subscales of avoidance $(\alpha=$ $.85)$, hyperarousal $(\alpha=.95)$, intrusions $(\alpha=.90)$ and negative cognitions $(\alpha=.89)$.

Resilience. The Brief Resilience Scale (BRS; Smith et al., 2008) is a six-item measure of resilience as a static trait, on a scale from 1 (strongly disagree) to 5 (strongly agree). This asks about everyday situations where resilience could be demonstrated, with questions such as, 'I tend to bounce back quickly after hard times'. A total score is obtained, with higher 
RUNNING HEAD: Traumatic stress, avoidant personality, resilience

scores indicative of greater resilience. The BRS demonstrates good test-re-test reliability and internal consistency (Smith, 2008) and showed good reliability in this study ( $\alpha=.89$ ).

Avoidant personality disorder traits. The Personality Diagnostic Questionnaire-4+ (PDQ-4+; Hyler, 1994) is a 99-item inventory that measures 12 different personality disorders. For the purpose of this study, only the seven questions relating to avoidant personality traits were used. Example items such as, 'I am more sensitive to criticism or rejection than other people' are rated as either true or false, with higher scores out of seven indicating the presence of AVPD characteristics. Although the intention of this study was not to apply diagnostic criteria, further clinical investigation is needed if four or more of the items are endorsed for the subscale; less than half of participants (48.1\%) marked 'true' for four or more responses. The PDQ-4+ has suitable psychometric properties (Hyler, 1994) and the avoidant items demonstrated good internal consistency $(\alpha=.82)$ in this study.

\section{Procedure}

Potential participants were provided with a generic link to an online survey, via an advertisement message shared on several social media sites and on posters across the university campus. The survey link invited participants to complete a series of measures relating to ACEs, resilience, personality traits and PTS symptoms. All participants gave informed consent before taking part in the study and could withdraw at any point. None of the participants were given an incentive to take part in the research. The study received ethical approval from the institutional ethics board.

\section{Data analysis}

Correlational analysis was undertaken to establish the strength and direction of relationships between the key study variables. Next, mediation analysis was conducted with the SPSS PROCESS macro (Hayes, 2013) to determine direct ( $\left.c^{\prime}\right)$ and indirect effects $(a b)$ 
RUNNING HEAD: Traumatic stress, avoidant personality, resilience

using 5,000 bootstrapped samples and bias-corrected 95\% confidence intervals (BCa CI).

Confidence intervals that do not contain zero indicate a significant indirect effect. The analysis of indirect effects is advantageous over traditional casual steps approaches as it does not require all paths in the mediation model to reach significance. To achieve the study aims, the number of ACEs was entered as the independent variable (X), overall PTS symptoms as the mediator variable (M), and AVPD and resilience as outcomes (Y) in two separate mediation models. Additional analyses of indirect effects with the four PCL-5 dimensions entered simultaneously as mediators are also reported.

In line with current recommendations (Hayes, 2013), unstandardized regression coefficients, direct and indirect effects are reported. Additionally, completely standardized indirect effect sizes $\left(a b_{c s}\right)$ allow for the identification of small $\left(a b_{c s} \leq .08\right)$, medium $\left(a b_{c s}\right.$ of .09 to .24$)$, or large $\left(a b_{c s} \geq .25\right)$ effects (Hayes, 2013). While the specification and directionality of the relationships within the models was driven by theory, reverse mediation analysis were also conducted in line with recommendations for cross-sectional models of indirect effects (Preacher \& Hayes, 2004). However, no significant findings or indirect effects were observed, thus confirming the hypothesised direction of the mediation models.

\section{Results}

\section{Preliminary analyses}

Descriptive and correlational data for ACEs, AVPD traits, resilience and PTS symptoms is reported in Table 2. The total number of ACEs experienced and AVPD traits were positively and moderately associated with PTS symptoms and the four dimensions assessed on the PCL-5. As expected, the four dimensions of PTS symptoms were positively and strongly correlated with one another. Resilience was negatively and moderately related to PTS symptoms and its four dimensions. The AVPD traits and resilience outcomes were 
RUNNING HEAD: Traumatic stress, avoidant personality, resilience

negatively and moderately correlated with one another, and unrelated to the number of ACEs experienced.

\section{[INSERT TABLE 2 ABOUT HERE]}

\section{Mediation analysis}

The full results of the mediation analysis are presented in Table 3. In relation to the first hypothesis, the mediation model with total number of ACEs as the independent variable and PTS symptoms as the mediator explained $28.1 \%$ of the variance in AVPD traits $[F(2$, $174)=33.96, p<.001]$. The direct effect of ACEs on AVPD traits was significant $(p<.001)$. In addition, PTS symptoms exerted significant and large positive indirect effects on AVPD traits $\left(a b_{c s}=.32\right.$; BCa CI: .22, .42), and thus mediated the association between ACEs and AVPD traits.

In relation to the second hypothesis, the mediation model with total number of ACEs as the independent variable and PTS symptoms as the mediator explained $27.1 \%$ of the variance in resiliency scores $[F(2,174)=32.36, p<.001]$. The direct effect of ACEs on resilience was significant $(p=.004)$, in addition to the significant and large negative indirect effect of PTS symptoms on the relationship between ACEs and resilience $\left(a b_{c s}=-.31 ; \mathrm{BCa}\right.$ CI: -.40, -.22). PTS symptoms therefore mediated the association between the number of ACEs and resilience.

Further analysis was conducted on the four dimensions of the PCL-5, with avoidance, hyperarousal, intrusions and negative cognitions entered into multiple mediation models distinct from the overall PTS symptoms mediator due to the overlap in these constructs. In the model with AVPD traits as the dependent variable, the four dimensions accounted for $30.4 \%$ of the variance in AVPD scores $[F(5,171)=15.00, p<.001]$. The direct effects of avoidance $(p=.012)$ and hyperarousal $(p=.012)$ on AVPD traits were significant, as were 
RUNNING HEAD: Traumatic stress, avoidant personality, resilience

their indirect effects (avoidance: $a b_{c s}=.13 ; \mathrm{BCa}$ CI: $.03, .27$; hyperarousal: $a b_{c s}=.17$; $\mathrm{BCa}$ CI: .04, .30). When resilience was entered as the dependent variable, only the direct effect of intrusions was significant $(p=.004)$, along with a significant negative indirect effect $\left(a b_{c s}=-\right.$ .11 ; BCa CI: -.22, -.02). Avoidance and hyperarousal thus mediated the relationship between the number of ACEs and AVPD traits, while intrusions mediated the association between the number of ACEs and resilience.

\section{[INSERT TABLE 3 ABOUT HERE]}

\section{Discussion}

While existing studies have explored PTS symptoms (e.g. Elklit et al., 2014), resilience (e.g. Campbell-Sills et al., 2009) and AVPD traits (e.g. Klein et al., 2015) as outcomes of the struggle with ACEs, less research has focused on the extent to which resilience and AVPD traits can be explained by the presence and intensity of PTS symptoms. Thus, this study aimed to investigate PTS symptoms as a mechanism whereby some people may report more (or less) AVPD traits and resiliency than others following ACEs. The results indicated that PTS symptoms explained approximately a quarter of the variance in AVPD traits and resilience, and therefore appear to have an important role in determining negative and positive psychological outcomes following ACEs.

Broadly, the results of this study are consistent with those in the wider literature relating to the detrimental impact of multiple ACEs on psychological functioning (Bellis et al., 2014; Finkelhor et al., 2011), such that as the frequency and magnitude of ACEs increases, so does symptom complexity (Schalinski et al., 2016). Given that survivors still endorsed PTS symptoms in adulthood, the findings attest to the significance of the timing of adverse events on PTS symptoms in later life (Dunn et al., 2017). The study therefore accounted for the developmental impact of multiple ACES on PTS symptoms, which in itself 
RUNNING HEAD: Traumatic stress, avoidant personality, resilience

goes beyond existing findings that tend to focus on responses to a single type of ACE only (Goodman et al., 2017). An additional implication of the current study is that it identifies that PTS symptoms arising from ACEs have a significant role to play in psychological adjustment, alongside the characteristics of the events themselves. This was evidenced by weaker direct effects from ACEs towards AVPD traits and resilience, compared to the indirect associations accounted for by PTS symptoms. It would appear that internal psychological states and responses to events matter more than the characteristics of the ACEs themselves.

\section{Mediating role of posttraumatic stress on negative and positive psychological outcomes}

This study draws attention to the indirect relationship between ACEs and AVPD traits, which, unlike BPD, has not yet received any significant attention in the literature. Indeed, much has been written about the role of adversity in BPD (e.g. Ball \& Links, 2009; MacIntosh et al., 2015), although the current findings also implicate ACEs and PTS symptoms as antecedents of AVPD traits. While this study supports the finding that more frequent ACEs are positively associated with AVPD traits (Hageman et al., 2015), the results also indicate that the same traits can be explained by PTS symptoms, and in particular, the avoidance and hyperarousal dimensions, following ACEs. It has been argued (WelanderVaten et al., 2018) that there is significant overlap between the anxieties experienced by PTS sufferers and those with AVPD, and so it is perhaps unsurprising that PTS symptoms are positively related to AVPD traits. Within this, the finding that avoidance mediated the relationship between the number of ACEs and AVPD traits is also expected given the overlap between this construct and the features of AVPD (Welander-Vaten et al., 2018). The results could therefore offer support to the 'betrayal trauma' hypothesis (Freyd, 1994), which proposes that avoidance - a defining characteristic of AVPD traits - is a function of attempts to dissociate from the abuse perpetrated by caregivers, and consequent anxiety surrounding 
RUNNING HEAD: Traumatic stress, avoidant personality, resilience

rejection and negative evaluations. Related to this, the significant mediation result for hyperarousal on AVPD traits may be linked to heightened arousal states which is a core feature of both PTS and AVPD (Lampe \& Malhi, 2018; Rettew et al., 2003).

Unlike the majority of the ACEs literature, this study drew attention to the potential for people to experience positive psychological changes. A direct relationship between the number of ACEs and resilience was found, which may not be surprising given that many people are resilient in the face of cumulative adverse events (Seery et al., 2010). However, this study uniquely found that levels of resilience were also accounted for by self-reported PTS symptoms and intrusive thoughts following ACEs. To date, studies have typically construed resilience as a buffer against distress (e.g. Lee et al., 2014; Pietrzak et al., 2010). While this effect was possibly illustrated by way of the negative relationship between PTS symptoms and resilience, the lack of bidirectional association in the mediation analysis suggests that resilience may also be an outcome of the psychological struggle with ACEs (Olsson et al., 2015). The negative relationship between intrusions and resilience could indicate that active processing of the event is needed to bring about positive outcomes (Bensimon, 2012; Brooks et al., 2019), rather than as a failure to integrate memories of the experiences (Halligan et al., 2003). A reduction in intrusive symptoms may indicate that survivors have processed their adverse experiences and have improved psychological functioning. Thus, this study highlights the potential for positive changes that are often overlooked in ACE research.

\section{Limitations of study and suggestions for further study}

This study has some limitations that must be noted when interpreting the findings. While the ACE-IQ is used in research in this area (e.g. Bellis et al., 2014), it fails to fully capture the severity and complex nature of trauma. For example, a child may have 
RUNNING HEAD: Traumatic stress, avoidant personality, resilience

experienced chronic, violent sexual abuse for many years, yet score fairly low in terms of trauma on the ACE-IQ. In addition to this, the study only included adversity experienced within the home that was committed by a caregiver or household member, which may not fully capture the range of ACEs that individuals may encounter. Although reverse mediation analyses were conducted to support the directionality of the relationships between variables, the cross-sectional nature of the study design precludes any conclusions in terms of causality. Longitudinal research is needed to confirm the nature and direction of relationships between ACEs, AVPD traits, resilience and PTS symptoms. The study also relied on retrospective and self-report accounts of ACEs by participants, which may be subject to under or overreporting and memory fallibility. Finally, while the study used a non-clinical sample, there were relatively high rates of ACE exposure, with many participants meeting clinical thresholds for PTSD and AVPD traits. Future studies should replicate the study in clinical samples to see if similar results are obtained.

\section{Clinical implications}

Importantly, this study's findings raise awareness among practitioners that people can report positive as well as negative changes following ACEs. In keeping with recent literature (e.g. Bensimon, 2012; Brooks et al., 2018), clinicians should be encouraged to consider the potential adaptive quality of PTS symptoms in that they not only serve as a marker for distress, but are also likely indicative of natural attempts to understand the impact of ACEs. The findings may point to further assessment of the valence of one's thoughts about their experiences, which may identify those at greatest risk of developing AVPD traits, and those who may demonstrate more resilience in the face of early life adversity.

It is also noteworthy that while people are remarkably resilient despite overwhelming adversity (Seery et al., 2010), the findings indicate the potential ameliorative effects of 
RUNNING HEAD: Traumatic stress, avoidant personality, resilience

resilience on PTS symptoms. Practitioners should focus efforts on enhancing resilience as a means to buffer against distress following ACEs that in turn may promote improvements to psychological wellbeing. Furthermore, the finding that PTS symptoms increase the likelihood of AVPD traits infers that treatments for this disorder should be trauma-informed. In this manner, clinicians should be wary of pathologising disordered personality traits without first considering whether experiences of childhood adversity and subsequent reactions to this are also pertinent.

\section{Conclusion}

PTS symptoms appear to be an important factor in the development of negative and positive changes following ACEs. Addressing gaps in prior research, the study identified trauma symptoms as the indirect pathway that explains AVPD traits after significant life events in childhood. At the same time, and somewhat paradoxically, PTS symptoms can also promote resilient outcomes. Trauma-informed interventions should be mindful of the multifaceted nature of PTS symptoms on psychological development, so that support can be tailored to enhance resilience among ACE survivors. 
RUNNING HEAD: Traumatic stress, avoidant personality, resilience

\section{References}

Auslander, W., Tlapek, S. M., Threlfall, J., Edmond, T., \& Dunn, J. (2018). Mental health pathways linking childhood maltreatment to interpersonal revictimization during adolescence for girls in the child welfare system. Journal of Interpersonal Violence, 33, 1169-1191. doi: 10.1177/0886260515614561

Ball, J. S., \& Links, P. S. (2009). Borderline personality disorder and childhood trauma: Evidence for a causal relationship. Current Psychiatry Reports, 11, 63-68. doi: 10.1007/s11920-009-0010-4

Banyard, V., Hamby, S., \& Grych, J. (2017). Health effects of adverse childhood events: Identifying promising protective factors at the intersection of mental and physical well-being. Child Abuse \& Neglect, 65, 88-98. doi: 10.1016/j.chiabu.2017.01.011

Békés, V., Beaulieu-Prévost, D., Guay, S., Belleville, G., \& Marchand, A. (2018). Traumarelated negative cognitions mediate the relationship between avoidant personality beliefs and impeded response to psychotherapy for PTSD. Journal of Aggression, Maltreatment \& Trauma, 1-16. doi: 10.1080/10926771.2018.1500504

Bellis, M. A., Hardcastle, K., Ford, K., Hughes, K., Ashton, K., Quigg, Z., \& Butler, N. (2017). Does continuous trusted adult support in childhood impart life-course resilience against adverse childhood experiences-a retrospective study on adult healthharming behaviors and mental well-being. BMC Psychiatry, 17, 110. doi: $10.1186 / \mathrm{s} 12888-017-1260-\mathrm{Z}$

Bellis, M. A., Lowey, H., Leckenby, N., Hughes, K., \& Harrison, D. (2014). Adverse childhood experiences: Retrospective study to determine their impact on adult health 
RUNNING HEAD: Traumatic stress, avoidant personality, resilience

behaviors and health outcomes in a UK population. Journal of Public Health, 36, 8191. doi: 10.1093/pubmed/fdt038

Bensimon, M. (2012). Elaboration on the association between trauma, PTSD and posttraumatic growth: The role of trait resilience. Personality and Individual Differences, 52, 782-787. doi: 10.1016/j.paid.2012.01.011

Bonanno, G. A. (2004). Loss, trauma, and human resilience: Have we underestimated the human capacity to thrive after extremely aversive events? American Psychologist, 59, 20-28. doi: 10.1037/0003-066X.59.1.20

Bovin, M. J., Marx, B. P., Weathers, F. W., Gallagher, M. W., Rodriguez, P., Schnurr, P. P., \& Keane, T. M. (2016). Psychometric properties of the PTSD Checklist for Diagnostic and Statistical Manual of Mental Disorders-Fifth Edition (PCL-5) in veterans. Psychological Assessment, 28, 1379-1391. doi: 10.1037/pas0000254

Bovin, M. J., Wolf, E. J., \& Resick, P. A. (2017). Longitudinal associations between posttraumatic stress disorder severity and personality disorder features among female rape survivors. Frontiers in Psychiatry, 8. doi: 10.3389/fpsyt.2017.00006

Brooks, M., Graham-Kevan, N., Robinson, S., \& Lowe, M. (2019). Trauma characteristics and posttraumatic growth: The mediating role of avoidance coping, intrusive thoughts, and social support. Psychological Trauma: Theory, Research, Practice and Policy, 11, 232-238. doi: 10.1037/tra0000372

Campbell-Sills, L., Forde, D. R., \& Stein, M. B. (2009). Demographic and childhood environmental predictors of resilience in a community sample. Journal of Psychiatric Research, 43, 1007-1012. doi: 10.1016/j.jpsychires.2009.01.013 
RUNNING HEAD: Traumatic stress, avoidant personality, resilience

Carlson, E. B., Palmieri, P. A., Field, N. P., Dalenberg, C. J., Macia, K. S., \& Spain, D. A. (2016). Contributions of risk and protective factors to prediction of psychological symptoms after traumatic experiences. Comprehensive Psychiatry, 69, 106-115. doi: 10.1016/j.comppsych.2016.04.022

Chapman, D. P., Whitfield, C. L., Felitti, V. J., Dube, S. R., Edwards, V. J., \& Anda, R. F. (2004). Adverse childhood experiences and the risk of depressive disorders in adulthood. Journal of Affective Disorders, 82, 217-225. doi: 10.1016/j.jad.2003.12.013

Cook, A., Spinazzola, J., Ford, J., Lanktree, C., Blaustein, M., Cloitre, M., \& Mallah, K. (2017). Complex trauma in children and adolescents. Psychiatric Annals, 35, 390398. doi: 10.3928/00485713-20050501-05

Crouch, E., Radcliff, E., Strompolis, M., \& Srivastav, A. (2019). Safe, stable, and nurtured: Protective factors against poor physical and mental health outcomes following exposure to adverse childhood experiences (ACEs). Journal of Child \& Adolescent Trauma, 12(2), 165-173. doi:10.1007/s40653-018-0217-9

Domhardt, M., Münzer, A., Fegert, J. M., \& Goldbeck, L. (2015). Resilience in survivors of child sexual abuse: A systematic review of the literature. Trauma, Violence, \& Abuse, 16, 476-493. doi: 10.1177/1524838014557288

Dunn, E. C., Nishimi, K., Powers, A., \& Bradley, B. (2017). Is developmental timing of trauma exposure associated with depressive and post-traumatic stress disorder symptoms in adulthood? Journal of Psychiatric Research, 84, 119-127. doi: 10.1016/j.jpsychires.2016.09.004 
RUNNING HEAD: Traumatic stress, avoidant personality, resilience

Elklit, A., Christiansen, D. M., Palic, S., Karsberg, S., \& Eriksen, S. B. (2014). Impact of traumatic events on posttraumatic stress disorder among Danish survivors of sexual abuse in childhood. Journal of Child Sexual Abuse, 23, 918-934. doi:

$10.1080 / 10538712.2014 .964440$

Felitti, V. J., Anda, R. F., Nordenberg, D., Williamson, D. F., Spitz, A. M., Edwards, V., \& Marks, J. S. (1998). Relationship of childhood abuse and household dysfunction to many of the leading causes of death in adults: The Adverse Childhood Experiences (ACE) Study. American Journal of Preventive Medicine, 14, 245-258. doi: $10.1016 / \mathrm{S} 0749-3797(98) 00017-8$

Finkelhor, D., Shattuck, A., Turner, H. A., Ormrod, R., \& Hamby, S. L. (2011). Polyvictimization in developmental context. Journal of Child \& Adolescent Trauma, 4, 291-300. doi: 10.1080/19361521.2011.610432

Freyd, J. J. (1994). Betrayal trauma: Traumatic amnesia as an adaptive response to childhood abuse. Ethics \& Behavior, 4, 307-329. doi: 10.1207/s15327019eb0404_1

Goodman, M. L., Gutarra, C., Billingsley, K. M., Keiser, P. H., \& Gitari, S. (2017). Childhood exposure to emotional abuse and later life stress among Kenyan women: a mediation analysis of cross-sectional data. Anxiety, Stress, \& Coping, 30, 469-483. doi: 10.1080/10615806.2016.1271876

Gratz, K. L., \& Tull, M. T. (2012). Exploring the relationship between posttraumatic stress disorder and deliberate self-harm: The moderating roles of borderline and avoidant personality disorders. Psychiatry Research, 199, 19-23. doi: 10.1016/j.psychres.2012.03.025 
RUNNING HEAD: Traumatic stress, avoidant personality, resilience

Hageman, T. K., Francis, A. J. P., Field, A. M., \& Carr, S. N. (2015). Links between childhood experiences and avoidant personality disorder symptomatology. International Journal of Psychology and Psychological Therapy, 15(1), 101-116.

Hagenaars, M. A., Fisch, I., \& van Minnen, A. (2011). The effect of trauma onset and frequency on PTSD-associated symptoms. Journal of Affective Disorders, 132, 192199. doi: 10.1016/j.jad.2011.02.017

Halligan, S. L., Michael, T., Clark, D. M., \& Ehlers, A. (2003). Posttraumatic stress disorder following assault: The role of cognitive processing, trauma memory, and appraisals. Journal of Consulting and Clinical Psychology, 71, 419-431. doi: 10.1037/0022006X.71.3.419

Happer, K., Brown, E. J., \& Sharma-Patel, K. (2017). Children's resilience and traumaspecific cognitive behavioral therapy: Comparing resilience as an outcome, a trait, and a process. Child Abuse \& Neglect, 73, 30-41. doi: 10.1016/j.chiabu.2017.09.021

Hayes, A. F. (2013). Introduction to mediation, moderation, and conditional process analysis: A regression-based approach. New York: Guilford Press.

Hu, T., Xu, S., \& Liu, W. (2018). A senior high school-based survey on the long-term impact of the Wenchuan earthquake on survivors' quality of life: PTSD as a mediator. Psychiatry Research, 270, 310-316. doi: 10.1016/j.psychres.2018.09.043

Hyler, S. E. (1994). PDQ-4+ Personality Diagnostic Questionnaire-4+. New York: New York State Psychiatric Institute.

Karam, E. G., Friedman, M. J., Hill, E. D., Kessler, R. C., McLaughlin, K. A., Petukhova, M., ... \& Girolamo, G. (2014). Cumulative traumas and risk thresholds: 12-month 
RUNNING HEAD: Traumatic stress, avoidant personality, resilience

PTSD in the World Mental Health (WMH) surveys. Depression and Anxiety, 31, 130142. doi: 10.1002/da.22169

Kim, J. S., Jin, M. J., Jung, W., Hahn, S. W., \& Lee, S. H. (2017). Rumination as a mediator between childhood trauma and adulthood depression/anxiety in non-clinical participants. Frontiers in Psychology, 8, 1597. doi: 10.3389/fpsyg.2017.0159

Klein, J. P., Roniger, A., Schweiger, U., Späth, C., \& Brodbeck, J. (2015). The association of childhood trauma and personality disorders with chronic depression: A cross-sectional study in depressed outpatients. The Journal of Clinical Psychiatry, 76, 794-801. doi: 0.4088/JCP. $14 \mathrm{~m} 09158$

Lee, J. S., Ahn, Y. S., Jeong, K. S., Chae, J. H., \& Choi, K. S. (2014). Resilience buffers the impact of traumatic events on the development of PTSD symptoms in firefighters. Journal of Affective Disorders, 162, 128-133. doi: 10.1016/j.jad.2014.02.031

MacIntosh, H. B., Godbout, N., \& Dubash, N. (2015). Borderline personality disorder: Disorder of trauma or personality, a review of the empirical literature. Canadian Psychology/Psychologie Canadienne, 56, 227-241. doi: 10.1037/cap0000028

Masten, A. S. (2001). Ordinary magic: Resilience processes in development. American Psychologist, 56, 227-238. doi: 10.1037/0003-066X.56.3.227

Neuner, F., Schauer, M., Karunakara, U., Klaschik, C., Robert, C., \& Elbert, T. (2004). Psychological trauma and evidence for enhanced vulnerability for posttraumatic stress disorder through previous trauma among West Nile refugees. BMC Psychiatry, 4, 34. doi: 10.1186/1471-244X-4-34 
RUNNING HEAD: Traumatic stress, avoidant personality, resilience

Olsson, L., Jerneck, A., Thoren, H., Persson, J., \& O’Byrne, D. (2015). Why resilience is unappealing to social science: Theoretical and empirical investigations of the scientific use of resilience. Science Advances, 1. doi: 10.1126/sciadv.1400217

Pechtel, P., Lyons-Ruth, K., Anderson, C. M., \& Teicher, M. H. (2014). Sensitive periods of amygdala development: the role of maltreatment in preadolescence. Neuroimage, 97, 236-244. doi: 10.1016/j.neuroimage.2014.04.025

Pietrzak, R. H., Johnson, D. C., Goldstein, M. B., Malley, J. C., Rivers, A. J., Morgan, C. A., \& Southwick, S. M. (2010). Psychosocial buffers of traumatic stress, depressive symptoms, and psychosocial difficulties in veterans of Operations Enduring Freedom and Iraqi Freedom: The role of resilience, unit support, and postdeployment social support. Journal of Affective Disorders, 120, 188-192. doi: 10.1016/j.jad.2009.04.015

Preacher, K. J., \& Hayes, A. F. (2004). SPSS and SAS procedures for estimating indirect effects in simple mediation models. Behavior Research Methods, 36, 717-731. doi: 10.3758/BF03206553

Rettew, D. C., Zanarini, M. C., Yen, S., Grilo, C. M., Skodol, A. E., Shea, M. T., ... \& Gunderson, J. G. (2003). Childhood antecedents of avoidant personality disorder: A retrospective study. Journal of the American Academy of Child \& Adolescent Psychiatry, 42, 1122-1130. doi: 10.1097/01.CHI.0000070250.24125.5F

Schalinski, I., Teicher, M. H., Nischk, D., Hinderer, E., Müller, O., \& Rockstroh, B. (2016). Type and timing of adverse childhood experiences differentially affect severity of PTSD, dissociative and depressive symptoms in adult inpatients. BMC Psychiatry, 16, 295. doi: 10.1186/s12888-016-1004-5 
RUNNING HEAD: Traumatic stress, avoidant personality, resilience

Seery, M. D., Holman, E. A., \& Silver, R. C. (2010). Whatever does not kill us: cumulative lifetime adversity, vulnerability, and resilience. Journal of Personality and Social Psychology, 99, 1025-1041. doi: 10.1037/a0021344

Sexton, M. B., Hamilton, L., McGinnis, E. W., Rosenblum, K. L., \& Muzik, M. (2015). The roles of resilience and childhood trauma history: Main and moderating effects on postpartum maternal mental health and functioning. Journal of Affective Disorders, 174, 562-568. doi: 10.1016/j.jad.2014.12.036

Smith, B. W., Dalen, J., Wiggins, K., Tooley, E., Christopher, P., \& Bernard, J. (2008). The Brief Resilience Scale: Assessing the ability to bounce back. International Journal of Behavioral Medicine, 15, 194-200. doi: 10.1080/10705500802222972

Stevens, N. R., Gerhart, J., Goldsmith, R. E., Heath, N. M., Chesney, S. A., \& Hobfoll, S. E. (2013). Emotion regulation difficulties, low social support, and interpersonal violence mediate the link between childhood abuse and posttraumatic stress symptoms. Behavior Therapy, 44, 152-161. doi: 10.1016/j.beth.2012.09.003

Suliman, S., Mkabile, S. G., Fincham, D. S., Ahmed, R., Stein, D. J., \& Seedat, S. (2009). Cumulative effect of multiple trauma on symptoms of posttraumatic stress disorder, anxiety, and depression in adolescents. Comprehensive Psychiatry, 50, 121-127. doi: 10.1016/j.comppsych.2008.06.006

Turner, H. A., Shattuck, A., Finkelhor, D., \& Hamby, S. (2017). Effects of poly-victimization on adolescent social support, self-concept, and psychological distress. Journal of Interpersonal Violence, 32, 755-780. doi: 10.1177/0886260515586376 
RUNNING HEAD: Traumatic stress, avoidant personality, resilience

Weathers, F. W., Litz, B. T., Keane, T. M., Palmieri, P. A., Marx, B. P., \& Schnurr, P. P. (2013). The PTSD Checklist for DSM-5 (PCL-5). Boston, MA: National Center for PTSD.

Weinbrecht, A., Schulze, L., Boettcher, J., \& Renneberg, B. (2016). Avoidant personality disorder: a current review. Current Psychiatry Reports, 18, 29. doi: 10.1007/s11920016-0665-6

Welander-Vaten, A., Torvik, F. A., Czajkowski, N., Kendler, K. S., Reichborn-Kjennerud, T., Knudsen, G. P., \& Ystrom, E. (2018). Relationships among avoidant personality disorder, social anxiety disorder, and normative personality traits: A twin study. Journal of Personality Disorders, 1-21. doi: 10.1521/pedi_2018_32_341

World Health Organisation (2012). Adverse Childhood Experiences - International Questionnaire (ACE-IQ) - rationale for ACE-IQ. Geneva: WHO. 
RUNNING HEAD: Traumatic stress, avoidant personality, resilience

\section{Tables}

Table 1.

Percentage of 21 adverse childhood experiences items reported by participants $(N=177)$.

\begin{tabular}{|c|c|c|c|c|}
\hline ACE-IQ items & Never & Once & $\begin{array}{l}\text { A few } \\
\text { times }\end{array}$ & $\begin{array}{l}\text { Many } \\
\text { times }\end{array}$ \\
\hline \multicolumn{5}{|l|}{ Parents/guardians/other household members... } \\
\hline $\begin{array}{l}\text { 1. Did not give me enough food when they could have } \\
\text { easily done so }\end{array}$ & 92.7 & 0.0 & 4.5 & 2.8 \\
\hline 2. Were too drunk or intoxicated to take care of me & 83.1 & 2.3 & 9.0 & 5.6 \\
\hline 3. Did not send me to school when it was available & 88.1 & 3.4 & 7.3 & 1.1 \\
\hline $\begin{array}{l}\text { 4. Yelled, screamed, sworn at, insulted or humiliated } \\
\text { me }\end{array}$ & 36.6 & 7.3 & 32.2 & 24.3 \\
\hline $\begin{array}{l}\text { 5. Threatened or abandoned me, or threw me out of } \\
\text { the house }\end{array}$ & 66.7 & 7.3 & 20.3 & 5.6 \\
\hline 6. Spanked, slapped, kicked, punched or beat me up & 80.8 & 6.8 & 10.2 & 2.3 \\
\hline 7. Hit me with a blunt or sharp object & 33.9 & 7.9 & 37.3 & 20.3 \\
\hline $\begin{array}{l}\text { 8. Touched me or made me touch them in a sexual } \\
\text { way when I did not want them to }\end{array}$ & 67.8 & 9.0 & 16.4 & 6.8 \\
\hline \multicolumn{5}{|l|}{$\begin{array}{l}\text { Saw/heard a parent/guardian/other household } \\
\text { member... }\end{array}$} \\
\hline 9. Being yelled, sworn at, insulted or humiliated at & 41.2 & 11.3 & 35.0 & 12.4 \\
\hline 10. Being slapped, kicked, punched or beaten up & 80.2 & 4.0 & 9.6 & 5.6 \\
\hline 11. Being hit or cut with an object & 79.1 & 6.8 & 6.2 & 7.3 \\
\hline \multicolumn{5}{|l|}{ How often... } \\
\hline 12. Were you bullied? & 20.3 & 13.6 & 44.6 & 21.5 \\
\hline 13. Were you in a physical fight? & 46.9 & 23.7 & 23.2 & 6.2 \\
\hline 14. Did you see or hear someone being beaten up? & 36.7 & 16.9 & 37.3 & 9.0 \\
\hline $\begin{array}{l}\text { 15. Did you see or hear someone being stabbed or } \\
\text { shot? }\end{array}$ & 85.9 & 9.6 & 4.0 & 0.0 \\
\hline \multirow[t]{2}{*}{$\begin{array}{l}\text { 16. Did you see or hear someone being threatened } \\
\text { with a knife or gun? }\end{array}$} & 74.6 & 15.3 & 9.0 & 0.6 \\
\hline & \multicolumn{2}{|c|}{ No } & \multicolumn{2}{|c|}{ Yes } \\
\hline \multicolumn{5}{|l|}{ Did you live with a household member who was... } \\
\hline $\begin{array}{l}\text { 17. A problem drinker, alcoholic, or misused street or } \\
\text { prescription drugs? }\end{array}$ & \multicolumn{2}{|c|}{81.4} & \multicolumn{2}{|c|}{18.6} \\
\hline 18. Depressed, mentally ill or suicidal? & \multicolumn{2}{|c|}{61.0} & \multicolumn{2}{|c|}{39.0} \\
\hline 19. Sent to jail or in prison? & \multicolumn{2}{|c|}{95.5} & \multicolumn{2}{|c|}{4.5} \\
\hline \multicolumn{5}{|l|}{ Did your... } \\
\hline 20. Parents ever separate or divorce? & \multicolumn{2}{|c|}{66.7} & \multicolumn{2}{|c|}{33.3} \\
\hline 21. Mother, father or guardian die? & \multicolumn{2}{|c|}{89.8} & \multicolumn{2}{|c|}{10.2} \\
\hline
\end{tabular}

Note. Percentages may not total $100 \%$ as figures are rounded. 
RUNNING HEAD: Traumatic stress, avoidant personality, resilience

\section{Table 2.}

Descriptive statistics and intercorrelations between key study variables $(N=177)$.

\begin{tabular}{|c|c|c|c|c|c|c|c|c|c|c|c|c|c|c|}
\hline & & $M$ & $S D$ & Range & Skew. & Kurt. & 1 & 2 & 3 & 4 & 5 & 6 & 7 & 8 \\
\hline 1. & Total number of ACEs & 16.92 & 10.57 & $1-21$ & .87 & .45 & - & & & & & & & \\
\hline 2. & AVPD traits & 3.36 & 2.34 & $0-7$ & .01 & -1.32 & .10 & - & & & & & & \\
\hline 3. & Resilience & 15.24 & 4.42 & $5-24$ & -.14 & -1.19 & -.09 & $-.53 * * *$ & - & & & & & \\
\hline 4. & Overall PTS symptoms & 20.54 & 17.89 & $0-63$ & .67 & -.78 & $.52 * * *$ & $.48 * * *$ & $-.49 * * *$ & - & & & & \\
\hline 5. & Avoidance & 5.48 & 5.19 & $0-17$ & .61 & -.96 & $.47 * * *$ & $.46^{* * *}$ & $-.42 * * *$ & $.74 * * *$ & - & & & \\
\hline 6. & Hyperarousal & 5.27 & 4.81 & $0-17$ & .73 & -.68 & $.55^{* * *}$ & $.45^{* * *}$ & $-.42 * * *$ & $.72 * * *$ & $.73 * * *$ & - & & \\
\hline 7. & Intrusions & 4.51 & 4.82 & $0-18$ & 1.00 & .10 & $.38 * * *$ & $.37 * * *$ & $-.48 * * *$ & $.74 * * *$ & $.74 * * *$ & $.68 * * *$ & - & \\
\hline 8. & Negative cognitions & 5.27 & 5.22 & $0-19$ & .79 & -.61 & $.46^{* * *}$ & $.45^{* * *}$ & $-.43 * * *$ & $.73 * * *$ & $.74 * * *$ & $.79 * * *$ & $.69 * * *$ & - \\
\hline
\end{tabular}

Note. ${ }^{*} p<.05, * * p<.01, * * * p<.001$. Skew = skewness; Kurt $=$ kurtosis. 
RUNNING HEAD: Traumatic stress, avoidant personality, resilience

Table 3.

Results of mediation analyses examining direct and indirect effects of number of ACEs on AVPD traits and resilience through PTS symptoms.

\begin{tabular}{|c|c|c|c|c|c|c|c|c|}
\hline \multirow[b]{2}{*}{$\begin{array}{l}\text { Independent } \\
\text { variable }(X)\end{array}$} & \multirow[b]{2}{*}{ Mediator $(M)$} & \multicolumn{4}{|c|}{ Direct effect $\left(c^{\prime}\right)$} & \multicolumn{2}{|c|}{ Indirect effect $(a b)$} & \multirow[b]{2}{*}{$\begin{array}{c}\text { Upper 95\% } \\
\text { BCa }\end{array}$} \\
\hline & & $b$ & $S E$ & $p$ & $b$ & Boot $S E$ & $\begin{array}{c}\text { Lower } 95 \% \\
\mathrm{BCa}\end{array}$ & \\
\hline & & \multicolumn{7}{|c|}{ AVPD traits $(Y)$} \\
\hline $\begin{array}{c}\text { Number of } \\
\text { ACEs }\end{array}$ & PTS symptoms & -.07 & .02 & .001 & .08 & .01 & .06 & .11 \\
\hline \multirow{4}{*}{$\begin{array}{c}\text { Number of } \\
\text { ACEs }\end{array}$} & Avoidance & .13 & .02 & $<.001$ & .03 & .02 & .01 & .07 \\
\hline & Hyperarousal & .15 & .06 & .012 & .03 & .02 & .01 & .08 \\
\hline & Intrusions & -.03 & .05 & .612 & -.01 & .01 & -.03 & .02 \\
\hline & Negative cognitions & .07 & .05 & .155 & .02 & .01 & -.01 & .04 \\
\hline & & \multicolumn{7}{|c|}{ Resilience $(Y)$} \\
\hline $\begin{array}{c}\text { Number of } \\
\text { ACEs }\end{array}$ & PTS symptoms & .11 & .04 & .004 & -.15 & .02 & -.20 & -.11 \\
\hline \multirow{4}{*}{$\begin{array}{c}\text { Number of } \\
\text { ACEs }\end{array}$} & Avoidance & -.06 & .09 & .555 & -.16 & .02 & -.07 & .03 \\
\hline & Hyperarousal & -.17 & .11 & .125 & -.05 & .04 & -.13 & .02 \\
\hline & Intrusions & -.28 & .09 & .004 & -.06 & .02 & -.11 & -.02 \\
\hline & Negative cognitions & -.10 & .10 & .334 & -.03 & .03 & -.08 & .03 \\
\hline
\end{tabular}

Notes. $S E=$ standard error; 95\% $\mathrm{BCa}=95 \%$ bias-corrected confidence interval; Boldface values indicate significant effects $(p<.05) ; c^{\prime}=$ unstandardized direct effects of the independent variable on the dependent variable $(X \rightarrow Y)$ controlling for the mediator; $a b=$ unstandardized indirect effects of the independent variable on the dependent variable via the mediator $(X \rightarrow M \rightarrow Y)$. 